\title{
LORENTZ GROUP LIE ALGEBRA MAP OF ULTRA-RELATIVISTIC RADIATING ELECTRON
}

\author{
H. Kawaguchi, K. Tsubota and T. Honma \\ Graduate School of Eng., Hokkaido Univ., Kita 13, Nishi 8, Kita-ku, Sapporo 060, Japan
}

\section{Abstract}

This paper discusses a precise numerical integrator for ultra-relativistic radiating electron motion. It is firstly shown that the covariant form Lorentz force equation of motion possesses the Lorentz group Lie algebra structure and the Lorentz-Dirac equation of motion as well. After that, a precise numerical integrator for radiating electron is constructed based on the Lie algebra properties. Numerical examples show that this integrator can be effectively used not only for the ultra-relativistic electron motion but also for a non-relativistic electron motions in pure magnetic field as well.

\section{INTRODUCTION}

One of the precise integrators for charged particle, the symplectic integrator, shows us its powerful properties for integration of the Hamilton systems. The leading principle for obtaining precise solutions is the conservation of the symplectic structure of the system. Here, we know that radiating electrons do not belong to the Hamilton systems when its velocity is very close to the light speed (ultra-relativistic velocity), because of radiation dumping effects. For that situation, any different kind leading principle are required, if one want to obtain precise solutions.

From this point of view, this paper considers of a numerical integrator for the ultra-relativistic radiating electron.

\section{LORENTZ GROUP LIE ALGEBRA PROPERTIES OF EQUATION OF MOTION}

The ordinary Lorentz force equation of motion (without the radiation reaction force) is expressed in the following covariant form (MKSA),

$$
m c \frac{d u^{\mu}}{d s}=e F_{\nu}^{\mu} u^{\nu}
$$

then one can find that the electromagnetic field tensor $F_{\nu}^{\mu}$ possesses special structure,

$$
F_{\nu}^{\mu}=\left(\begin{array}{cccc}
0 & E_{x} / c & E_{y} / c & E_{z} / c \\
E_{x} / c & 0 & B_{z} & -B_{y} \\
E_{y} / c & -B_{z} & 0 & B_{x} \\
E_{z} / c & B_{y} & -B_{x} & 0
\end{array}\right)
$$

or

$$
F_{\nu}^{\mu}=\frac{\mathbf{E}}{c} \bullet \mathbf{K}-\mathbf{B} \bullet \mathbf{S}
$$

where $\mathbf{K}$ and $\mathbf{S}$ are the boost and rotation operators, which construct one of irreducible representations of the Lorentz group Lie algebra[1],

$$
\begin{aligned}
& \mathbf{K}=\left(K_{x}, K_{y}, K_{z}\right), \\
& K_{x}=\left(\begin{array}{llll}
0 & 1 & 0 & 0 \\
1 & 0 & 0 & 0 \\
0 & 0 & 0 & 0 \\
0 & 0 & 0 & 0
\end{array}\right), K_{y}=\left(\begin{array}{llll}
0 & 0 & 1 & 0 \\
0 & 0 & 0 & 0 \\
1 & 0 & 0 & 0 \\
0 & 0 & 0 & 0
\end{array}\right), K_{z}=\left(\begin{array}{llll}
0 & 0 & 0 & 1 \\
0 & 0 & 0 & 0 \\
0 & 0 & 0 & 0 \\
1 & 0 & 0 & 0
\end{array}\right), \\
& \mathbf{S}=\left(S_{x}, S_{y}, S_{z}\right), \\
& S_{x}=\left(\begin{array}{cccc}
0 & 0 & 0 & 0 \\
0 & 0 & 0 & 0 \\
0 & 0 & 0 & -1 \\
0 & 0 & 1 & 0
\end{array}\right), S_{y}=\left(\begin{array}{cccc}
0 & 0 & 0 & 0 \\
0 & 0 & 0 & 1 \\
0 & 0 & 0 & 0 \\
0 & -1 & 0 & 0
\end{array}\right), S_{z}=\left(\begin{array}{cccc}
0 & 0 & 0 & 0 \\
0 & 0 & -1 & 0 \\
0 & 1 & 0 & 0 \\
0 & 0 & 0 & 0
\end{array}\right) \text {. }
\end{aligned}
$$

The representation (3) can be naturally understood if we remember that the four velocity vector $u^{\mu}$ is exactly confined by the following identity,

$$
u^{\nu} u_{\nu}=1
$$

that is to say, Eq. (1) merely tells us that the four velocity is governed by the Lorentz transformation along the particle trajectory and then the field tensor $F_{\nu}^{\mu}$ plays the role of the Lorentz group Lie algebra. (This situation can be expressed in Fig. 1. The terminal point of the four vector is always on unit sphere in $4 \mathrm{D}$ Minkowski space and its trajectory moves along "vector" $F_{\nu}^{\mu}$.)

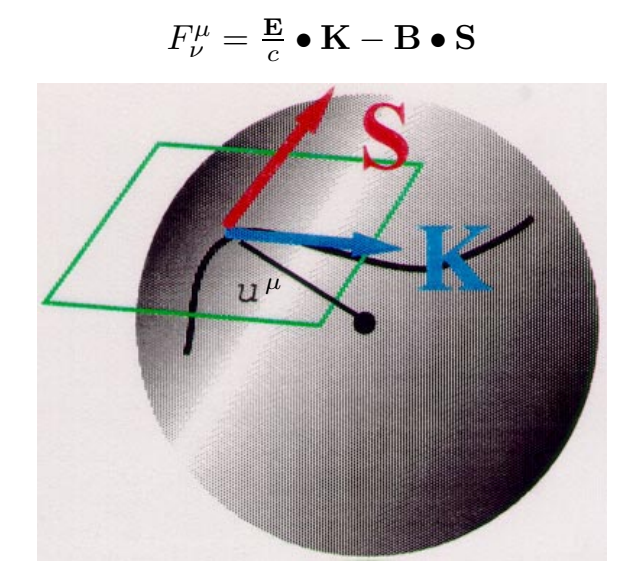

Figure 1: Trajectory of terminal point of four vector.

There also exists similar situation in the radiation dumping phenomena. The ultra-relativistic radiating electron is governed by the Lorentz-Dirac equation of motion [1, 2, 3],

$$
\begin{aligned}
m c \frac{d u^{\mu}}{d s}= & e F_{\nu}^{\mu} u^{\nu} \\
& +\frac{e^{2}}{6 \pi \epsilon_{0} c}\left(\frac{d^{2} u^{\mu}}{d s^{2}} u_{\nu}-\frac{d^{2} u_{\nu}}{d s^{2}} u^{\mu}\right) u^{\nu},
\end{aligned}
$$


Then, the radiation reaction term (the second term) also has similar structure as the field tensor and can be expressed by using the irreducible representations, $\mathbf{K}$ and $\mathbf{S}$,

$$
m c \frac{d u^{\mu}}{d s}=e H_{\nu}^{\mu} u^{\nu}
$$

where

$$
\begin{aligned}
H_{\nu}^{\mu} & \equiv F_{\nu}^{\mu}+G_{\nu}^{\mu} \\
& \equiv F_{\nu}^{\mu}+\frac{e}{6 \pi \epsilon_{0} c}\left(\frac{d^{2} u^{\mu}}{d s^{2}} u_{\nu}-\frac{d^{2} u_{\nu}}{d s^{2}} u^{\mu}\right)
\end{aligned}
$$

\section{CONSTRUCTION OF PRECISE INTEGRATOR FOR RADIATING ELECTRON}

The foregoing discussion tells us that the four velocity vector $u^{\mu}$ satisfies,

$$
u^{\mu}(s)=\exp \left(\frac{e}{m c} F_{\nu}^{\mu} s\right) u^{\nu}(0)
$$

That is to say, the exponential fuction is operating on $u^{\mu}$ as the Lorentz transformation. Then, one more integral of Eq. (10) yields the following transformation rule of the four dimensional particle coordinates $x^{\mu}$,

$$
x^{\mu}(s)=x^{\nu}(0)+\int_{0}^{s} d \sigma \exp \left(\frac{e}{m c} F_{\nu}^{\mu} \sigma\right) u^{\nu}(0)
$$

Unifying Eqs. (10) and (11), we obtain the following transformation formula,

$$
\left[\begin{array}{l}
x^{\mu}(s) \\
u^{\mu}(s)
\end{array}\right]=\left[\begin{array}{cc}
1 & \int_{0}^{s} d \sigma \exp \left(\frac{e}{m c} F_{\nu}^{\mu} \sigma\right) \\
0 & \exp \left(\frac{e}{m c} F_{\nu}^{\mu} s\right)
\end{array}\right]\left[\begin{array}{l}
x^{\nu}(0) \\
u^{\nu}(0)
\end{array}\right]
$$

To be iteratively used for the infinitesimal interval $d s$, the transformation (12) gives us a numerical integral formulation which exactly utilizes the Lorenz transformation of $u^{\mu}$ along the particle trajectory.

And then, almost same formula is also obtained for the radiating electron,

$$
\left[\begin{array}{l}
x^{\mu}(s) \\
u^{\mu}(s)
\end{array}\right]=\left[\begin{array}{cc}
1 & \int_{0}^{s} d \sigma \exp \left(\frac{e}{m c} H_{\nu}^{\mu} \sigma\right) \\
0 & \exp \left(\frac{e}{m c} H_{\nu}^{\mu} s\right)
\end{array}\right]\left[\begin{array}{l}
x^{\nu}(0) \\
u^{\nu}(0)
\end{array}\right]
$$

\section{FORMULAS OF IRREDUCIBLE REPRESENTATION}

Before proceed to concrete numerical calculations, some useful formulas of the irreducible representations of the Lorentz group Lie algebra are summarized for later reference here. If we denote arbitrary unit vectors as $\mathbf{e}$ and $\mathbf{b}$, the following formula are satisfied,

$$
\begin{aligned}
(\mathbf{e} \bullet \mathbf{K})^{3} & =(\mathbf{e} \bullet \mathbf{K}) \\
(\mathbf{b} \bullet \mathbf{S})^{3} & =-(\mathbf{b} \bullet \mathbf{S}) \\
{\left[S_{i}, S_{j}\right] } & =\epsilon_{i j k} S_{k} \\
{\left[S_{i}, K_{j}\right] } & =\epsilon_{i j k} K_{k} \\
{\left[K_{i}, K_{j}\right] } & =-\epsilon_{i j k} S_{k}
\end{aligned}
$$

where $\epsilon_{i j k}$ denotes the Levi-Civita pseudotensor. Moreover, one can readily confirm the following identities too,

$$
\begin{aligned}
& (\mathbf{b} \bullet \mathbf{S})(\mathbf{e} \bullet \mathbf{K})(\mathbf{b} \bullet \mathbf{S})=0 \\
& (\mathbf{e} \bullet \mathbf{K})(\mathbf{b} \bullet \mathbf{S})(\mathbf{e} \bullet \mathbf{K})=0
\end{aligned}
$$

These formula can be effectively used in calculations of exponential function in the formulas (12) and (13).

\section{EXAMPLES OF NUMERICAL CALCULATION}

The first example is an electron motion in pure magnetic field, especially no radiation dumping case. In this case, the identity (6) shows us special aspect, i.e., the formula gives us numerical solutions which exactly satisfy the momentum conservation law. Adding to this, we find that the exponential function in Eqs. (10) and (11) can result in the following reduced expression,

$$
\begin{aligned}
u^{\mu}(s)= & {\left[1-(\mathbf{b} \bullet \mathbf{S}) \sin \left(\frac{e B}{m c} s\right)\right.} \\
& \left.+(\mathbf{b} \bullet \mathbf{S})^{2}\left(1-\cos \left(\frac{e B}{m c} s\right)\right)\right] u^{\mu}(0)
\end{aligned}
$$

which makes the calculation time much shorter (where $\mathbf{b}$ is normalized unit vector of $\mathbf{B}$ and $B$ is its absolute value). The position $x^{\mu}$ is also expressed in the same manner. The figure 2 shows a typical example, the 3D electron motion in so-called magnetic mirror profile. In Fig. 3, velocity changes during the motion, which are caused by numerical errors, are indicated for the ordinary Runge-Kutta method and presented one, respectively. The initial electron energy is taken to be $200 \mathrm{MeV}$. The simulation result shows us that the presented method indeed can exactly conserve the particle momentum.

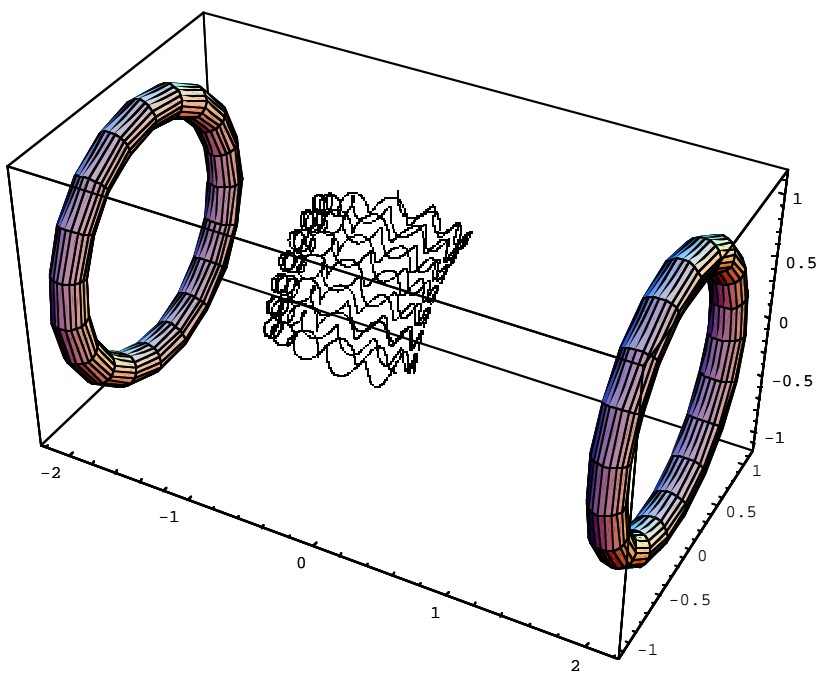

Figure 2: Electron motion in magnetic mirror profile $(200 \mathrm{MeV})$.

The next example is the main purpose of this paper, the ultra-relativistic radiating electron. An electron orbit, 


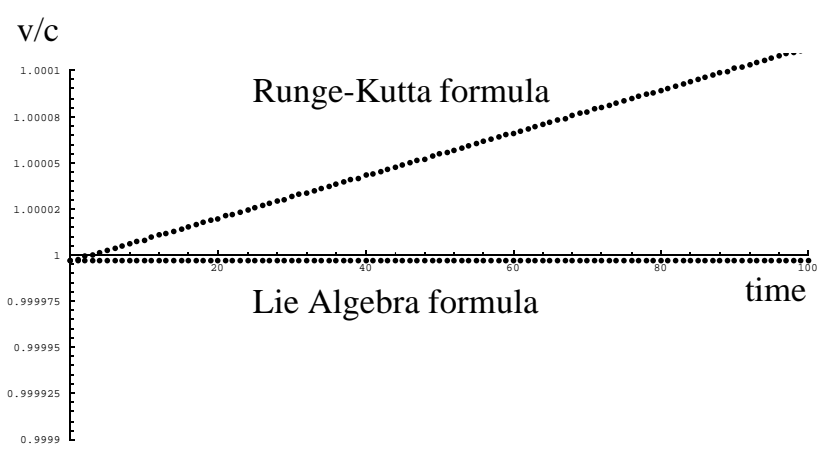

Figure 3: Velocity change caused by numerical error.

which has strong radiation dumping, is shown in Fig. 4. The initial electron energy is taken to be $500 \mathrm{GeV}$. In this case, the ordinary Runge-Kutta method cannot work because the calculation is very sensitive for numerical errors. On the other hand, the presented method still works well. The figure 5 shows the change of the Minkowski norm during the motion, which shows that the identity (6) exactly satisfied even in this energy region (non-Hamilton systems).

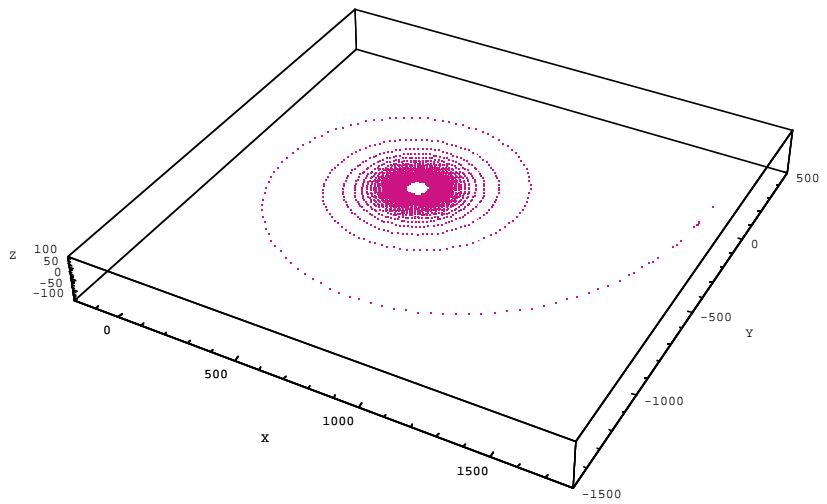

Figure 4: Ultra-relativistic radiating electron motion $(500 \mathrm{GeV})$.

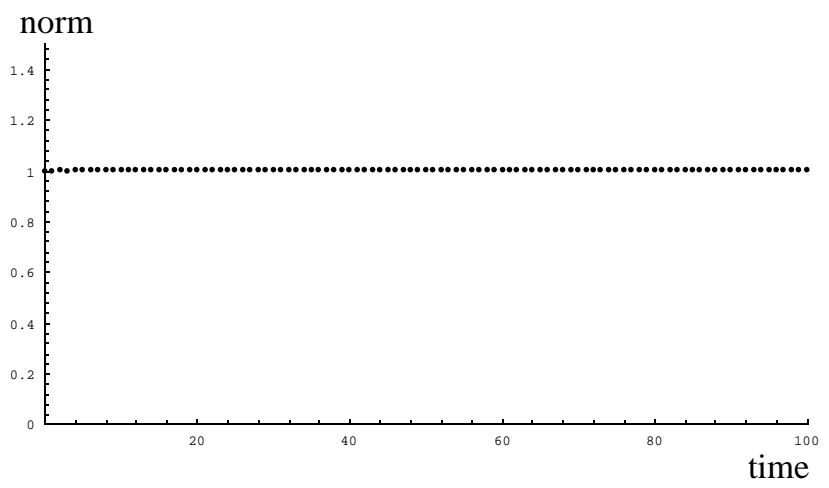

Figure 5: Change of Minkowski norm.

\section{SUMMARY}

This paper has presented a precise numerical integrator for the ultra-relativistic radiating electron based on the Lorentz group Lie algebra. Some simple numerical calculations show its validity. However, serious problem still exists in the ultra-relativistic electron orbit calculation. It is wellknown that the Lorentz-Dirac equation is itself approximation for the case of weak radiation dumping[3]. To fully treat strong radiation dumping some more modifications are required.

\section{REFERENCES}

[1] J.D. Jackson, Classical Electrodynamics, 2nd Ed., (Wiley, New York 1975).

[2] W.K.H. Panofsky and M. Phillips, Classical Electricity and Magnetism (Addison-Wesley, Cambridge 1961).

[3] L.D. Landau and E.M. Lifshitz, Classical Theory of Fields, (Pergamon, Oxford, 1971). 